Internist 2018 $59: 1-2$

https://doi.org/10.1007/s00108-017-0371-4

Online publiziert: 19. Dezember 2017

(c) Springer Medizin Verlag GmbH, ein Teil von Springer Nature 2017

CrossMark

\section{J. Mössner ${ }^{1} \cdot$ M. Reincke}

${ }^{1}$ Klinik und Poliklinik für Gastroenterologie und Rheumatologie, Department für Innere Medizin, Neurologie und Dermatologie, Universitätsklinikum Leipzig, AöR, Leipzig, Deutschland

${ }^{2}$ Medizinische Klinik und Poliklinik IV, Klinikum der Universität München, Ludwig-Maximilians-Universität München, München, Deutschland

\title{
Funktionsdiagnostik in der Inneren Medizin
}

Es mag trivial erscheinen: Im Studium haben wir die Anatomie erlernt, da die Morphologie die Grundlage bzw. Voraussetzung für die Funktion eines Organs schafft. Die Funktion eines Organs und sein Zusammenspiel mit anderen Organen haben wir in der Physiologie gelernt; die Funktion auf zellulärer Ebene in der Biochemie. Bei der Pathogenese vieler Erkrankungen in der Inneren Medizin führen pathologische Prozesse in der Epigenetik, Genetik und im zellulären Stoffwechsel zur gestörten Funktion der einzelnen Zelle, des gesamten Organs und auch des gesamten Organismus. Einer gestörten Funktion liegt oft auch eine pathologische Morphologie zugrunde.

\section{》) Die Innere Medizin ist diag- nostisch sehr "morphologisch" ausgerichtet}

Wenn letztlich die Funktionsfähigkeit der Zelle, des Organs, des Organismus für das Leben entscheidend ist, überrascht es, dass wir bezüglich der Diagnostik in der Inneren Medizin doch sehr „morphologisch“ ausgerichtet sind: Bildgebung (Sonographie, Endoskopie, konventionelle Röntgendiagnostik, Computertomographie, Magnetresonanztomographie, Nuklearmedizin etc.), Histologie, Makropathologie. Im Gegensatz zu früher bieten aber viele der bildgebenden Verfahren auch die Möglichkeit, zusätzlich die Funktion zu untersuchen. Mehr noch: Bildgebung entwickelt sich immer häufiger zur quantitativ erfassten Funktionsdiagnostik. Als Beispiele seien die Messung der Durchblutung mit der Duplexsonographie und die Bestimmung der Herzmuskelfunktion mit der Stressechokardiographie genannt. Letztlich misst auch die Fluordesoxyglukose-Positronenemissionstomographie eine Funktion, die Glukoseaufnahme der Tumorzelle. Wir glauben daher, dass eine Bestandsaufnahme des Stellenwerts der Funktionsdiagnostik in der Inneren Medizin 2018 gerechtfertigt ist. Leider konnten dabei aus Platzgründen nicht alle acht Schwerpunkte der Inneren Medizin berücksichtigt werden. Diese Ausgabe beschränkt sich auf die Funktionsdiagnostik in der Kardiologie, Pneumologie, Gastroenterologie, Endokrinologie und Nephrologie.

Herrmann, Kraus und Frantz aus Würzburg zeigen am Beispiel der Magnetresonanztomographie und der Echokardiographie, wie in der Kardiologie bildgebende Verfahren für die Funktionsdiagnostik des Herzens relevant sind. Das Elektrokardiogramm (EKG) gehört sicher $\mathrm{zu}$ den in der Inneren Medizin am längsten eingesetzten Verfahren zur Messung einer Funktion. Es ist erstaunlich, dass das Ruhe-EKG und die Blutdruckmessung nach Scipione Riva-Rocci über die Jahrzehnte nicht an Bedeutung verloren haben.

\section{》) Gerade in der Pneumologie ist die Funktionsdiagnostik unverzichtbar}

In der Pneumologie mag die Trennung zwischen Bildgebung (z. B. Bronchoskopie) und Funktionsuntersuchungen schärfer sein. Held, Baron und Jany aus
Würzburg stellen überzeugend dar, dass gerade in der Pneumologie die Funktionsdiagnostik, etwa in Form der Ganzkörperplethysmographie, unverzichtbar ist. So ist die Funktionsdiagnostik auch essenziell, um kardiale Ursachen einer eingeschränkten Lungenfunktion von rein pulmonalen zu differenzieren. $\mathrm{Na}$ türlich ist auch in der Pneumologie die Funktionsdiagnostik von entscheidender Bedeutung, wenn das Therapieansprechen beurteilt werden soll. Das Schlaflabor zur Beurteilung schlafbezogener obstruktiver Ventilationsstörungen ist in der Pneumologie unverzichtbar. So erfährt der Leser, wann eine Polygraphie und wann eine Polysomnographie durchzuführen ist.

Das Spektrum der Funktionsuntersuchungen in der Gastroenterologie ist breit gefächert. Während die Untersuchung der exokrinen Pankreasfunktion in der Diagnostik und bei der Therapieentscheidung an Bedeutung verloren hat, ist gerade vor einer Leberresektion oder palliativen Tumortherapie wie der selektiven internen Radiotherapie eine Abschätzung der Funktionsfähigkeit des verbleibenden Parenchyms entscheidend. Die Leberfunktionsdiagnostik konnte in dieser Übersicht leider aus Platzgründen nicht berücksichtigt werden. Hollenbach, Hoffmeister, Rosendahl und der Mitherausgeber Mössner aus Leipzig und Halle (Saale) geben einen Überblick über Routineverfahren, beispielsweise über die $\mathrm{H}_{2}$-Atemtests zur Beurteilung der Fragen Laktasemangel, bakterielle Fehlbesiedlung und Dünndarmpassagezeit oder den ${ }^{13} \mathrm{C}$-HarnstoffAtemtest zum Nachweis einer Helicobac- 
ter-pylori-Infektion. Vorgestellt werden auch die Verfahren zur Beurteilung der Ösophagus-, Magen- oder Dünndarmmotilität oder im Rahmen der häufigen Refluxerkrankung die Langzeit-pH-Metrie und die Impedanzmessung. Auch in der Gastroenterologie ist die Sonographie unverzichtbar für die Untersuchung von Funktionen wie Motilität oder Durchblutung.

Eine endokrinologische Diagnostik wäre ohne Beurteilung der Funktion undenkbar. Auch in der Endokrinologie ist das Spektrum weit: Nimmt ein Knoten der Schilddrüse Jodid auf? Auf welcher Ebene ist der Regelkreis aus Hypothalamus, Hypophyse und Nebenniere gestört? Auernhammer und der Mitherausgeber Reincke aus München stellen die Stimulationstests zur Abklärung einer vermuteten Unterfunktion und die Suppressionstests zur Sicherung einer möglichen Überfunktion vor. Präanalytische „caveats“ und Assay-abhängige Normwerte in der Diagnostik sind hierbei sorgfältig zu beachten. Die Beantwortung der Frage, ob eine „critical illness-related corticosteroid insufficiency" (CIRCI) vorliegt, ist lebensentscheidend, wie auch die Funktionsdiagnostik bei der Frage, ob bei einem hypertensiven Patienten ein Phäochromozytom vorliegt.

In ihrem Beitrag zur Nierenfunktionsdiagnostik machen Huynh-Do, Fiedler und Vogt aus Bern den Leser sicher schon im Titel neugierig: „Kreatinin ist nicht alles“. Tests zur Beurteilung der glomerulären Funktion sind sicher nicht ,alles“. Wir wollen in der Einführung die Neugier aufrechterhalten und wünschen eine gute Lektüre.

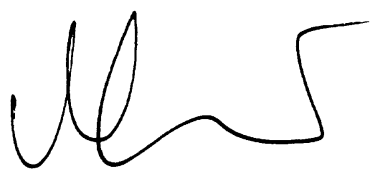

J. Mössner

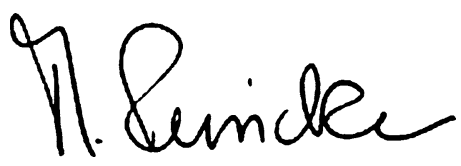

M. Reincke

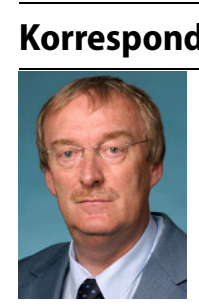

Vom Gen zur Therapie

Antisense-Technik bremst seltene neurologische Erkrankungen

Prof. Dr. J. Mössner

Klinik und Poliklinik

für Gastroenterologie

und Rheumatologie,

Department für Innere

Medizin, Neurologie

und Dermatologie,

Universitätsklinikum Leipzig,

AöR

Liebigstr. 20, 04103 Leipzig,

Deutschland

joachim.moessner@

medizin.uni-leipzig.de

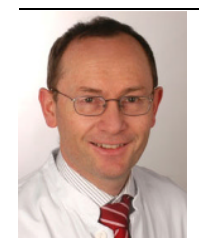

Prof. Dr. M. Reincke

Medizinische Klinik und Poliklinik IV, Klinikum der Universität München, Ludwig-MaximiliansUniversität München Ziemssenstr. 1, 80336 München, Deutschland martin.reincke@med.unimuenchen.de

Interessenkonflikt. J. Mössner und M. Reincke geben an, dass kein Interessenkonflikt besteht.
„Der Schlüssel zur Behandlung Seltener Erkrankungen liegt oft in ihren genetischen Ursachen", sagt Professor Christine Klein, Neurogenetikerin aus Lübeck und Stellvertretende Präsidentin der Deutschen Gesellschaft für Neurologie (DGN).

Mit der Einführung des "next generation sequencing" konnten die Aufklärungs raten bei Patienten mit seltenen erblichen Erkrankungen in den letzten Jahren von 5 auf circa $40 \%$ erhöht werden.

\section{Antisense-Therapie}

Kürzlich erhielt das Medikament Nusinersen zur Behandlung der Spinalen Muskelatrophie (SMA) die europäische Zulassung - ein Durchbruch für die neurologische Forschung, denn gegen die seltene schwere Erbkrankheit gab es zuvor keine Therapieoption. Das Medikament verlangsamt das Fortschreiten der Erkrankung. Die neuromuskuläre Erbkrankheit betrifft vor allem Kinder. Aufgrund eines Gendefekts gehen Nervenzellen zugrunde, die Bewegungen kontrollieren, weshalb die Muskulatur, einschließlich der Schluckund Atemmuskulatur, degeneriert.

Die Antisense-Technik ist auch für andere seltene Erbkrankheiten eine vielversprechende Option. Derzeit laufen Therapiestudien bei der Huntington-

Krankheit. Für die Muskeldystrophie Typ Duchenne ist bereits eine Therapie auf Basis von Antisense-Nukleotiden zugelassen, woraus sich erstmals eine kausale Therapieoption ergibt.

Literatur:

Klein CJ, Foroud TM. Neurology Individualized Medicine: When to Use Next-Generation Sequencing Panels. Mayo Clin Proc. 2017 92(2):292-305. 10.1016/j.mayocp.2016.09.008 Maeder ML, Gersbach CA. Genomeediting Technologies for Gene and Cell Therapy. Mol Ther. 2016 24(3):430-46. 10.1038/mt.2016.10

Prof. Dr. med. Hans-Christoph Diener, Pressesprecher der DGN 